\title{
Management of diabetic macular oedema: new insights and global implications of DRCR protocol V
}

\author{
Ning Cheung ${ }^{1,2} \cdot$ Chiu Ming Gemmy Cheung ${ }^{1,2} \cdot$ Steven James Talks ${ }^{3} \cdot$ Tien Yin Wong $\mathbb{D}^{1,2}$
}

Received: 28 October 2019 / Accepted: 11 November 2019 / Published online: 16 December 2019

(c) The Royal College of Ophthalmologists 2019

Over 400 million people have diabetes worldwide, with the number projected to increase close to 650 million in the next two decades [1]. In the United Kingdom alone, there are over 4 million people with diabetes [2]. As the most common microvascular complication of diabetes, diabetic retinopathy (DR) is therefore expected to remain as a global threat to vision and economy [3, 4].

Diabetic macular oedema (DMO) is now the most prevalent vision-threatening form of DR, particularly among adults with type 2 diabetes [4]. The global prevalence of DMO has been estimated to be $6.8 \%$. Thus, about 27 million adults are affected by DMO worldwide. Fortunately, since 2010, the advent of ocular anti-vascular-endothelial-growth-factor (VEGF) therapy has greatly improved visual prognosis for patients with vision loss due to centre-involved DMO [5]. It has become the standard-of-care treatment for these patients in many developed countries [4, 6-9].

Besides DMO, ocular anti-VEGF therapy has similarly shown clear benefits for other retinal angiogenic diseases, such as proliferative DR, neovascular age-related macular degeneration, and macular oedema secondary to retinal vein occlusion [10, 11]. Its widespread use over the last two decades has undoubtedly saved sight for millions of patients worldwide. However, increasing evidence for the expanding indications of anti-VEGF therapy have also created tremendous stress to the healthcare system. Many countries are struggling to cope with the continually rising number of patients requiring this, often regular and long-term,

Tien Yin Wong

wong.tien.yin@singhealth.com.sg

1 Singapore Eye Research Institute, Singapore National Eye Centre, Singapore, Singapore

2 Duke-NUS Medical School, National University of Singapore, Singapore, Singapore

3 Newcastle Upon Tyne Hospitals NHS Foundation Trust, Newcastle upon Tyne, UK treatment. Furthermore, this treatment burden could be compounded by clinicians who might be tempted to use the current body of evidence to assume its efficacy beyond proven clinical indications [12]. One such questionable indication was centre-involved DMO with good vision. This is an increasing issue, especially in areas with good DR screening, access to anti-VEGF therapy and increased use of optical coherence tomography in referral pathways [13]. Recently published data from the DRCR network aimed to address this important issue [14].

In the DRCR Protocol V study, three different management strategies for centre-involved DMO with good vision (visual acuity 20/25 or better) were compared in a large, adequately powered randomised clinical trial [14]. After 2 years of follow-up, there was no observable difference in visual outcome among the 702 patients who were initially managed with either aflibercept, laser or observation, with a $\geq 5$-letter decrease in 16\%, 17\% and 19\% respectively. Aflibercept was used when vision worsened in the laser $(25 \%)$ and observation (34\%) cohorts. Compared to eyes in the observation group, eyes in the laser group had a smaller chance (10\% less absolute likelihood or $34 \%$ less relative likelihood) of requiring aflibercept injections. The mean visual acuity at 2 years was 20/20 in all three cohorts, and the proportions of eyes with this level of vision was $77 \%$ in the aflibercept group, $71 \%$ in the laser group, and $66 \%$ in the observation group. Collectively, these data suggest that it is generally safe to initially observe centre-involved DMO with good vision, and consider treatment only when visual impairment occurs.

Although the results of the Protocol V may be unsurprising, the study does illustrate the importance of identifying whom not to treat in the era of increasing use of anti-VEGF therapy for proven and unproven indications. These data allow clinicians to feel safe to adopt a more conservative approach in the management of patients with centre-involved DMO whose vision is not yet affected (Fig. 1). As the Early Treatment Diabetic Retinopathy Study showed almost 3 decades ago, about $40 \%$ of eyes with 
Fig. 1 A clinical case of 62 year man with centre-involved diabetic macular oedema and good visual acuity (VA) remaining stable under observation over 2 years. Although macular oedema appears to be worsening in the right eye but improving in the left eye, VA remained stable in both eyes.
First Examination - January 2017

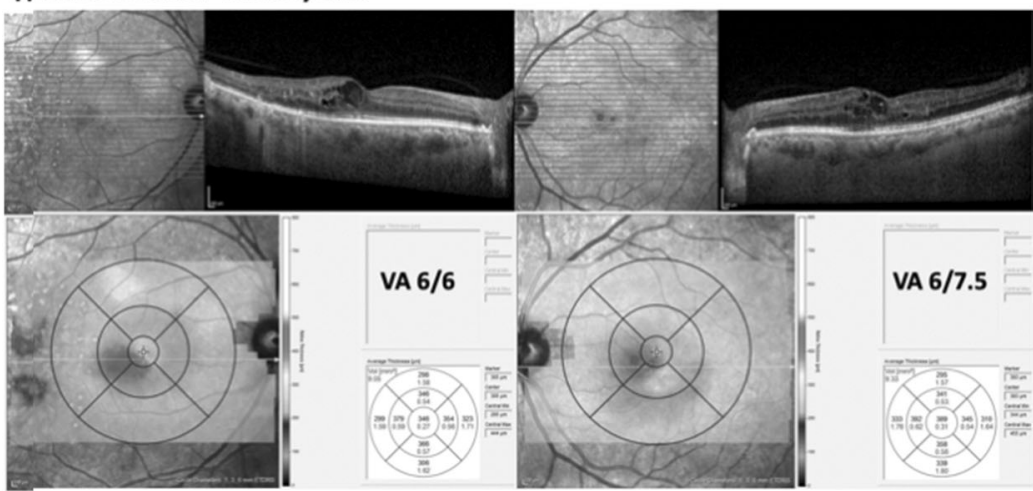

Follow-up Examination - August 2017

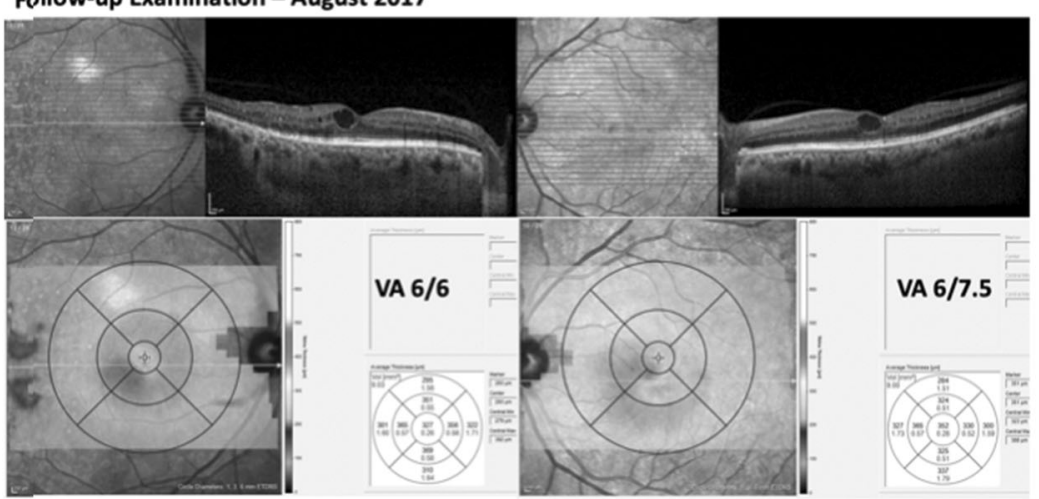

Follow-up Examination - October 2018

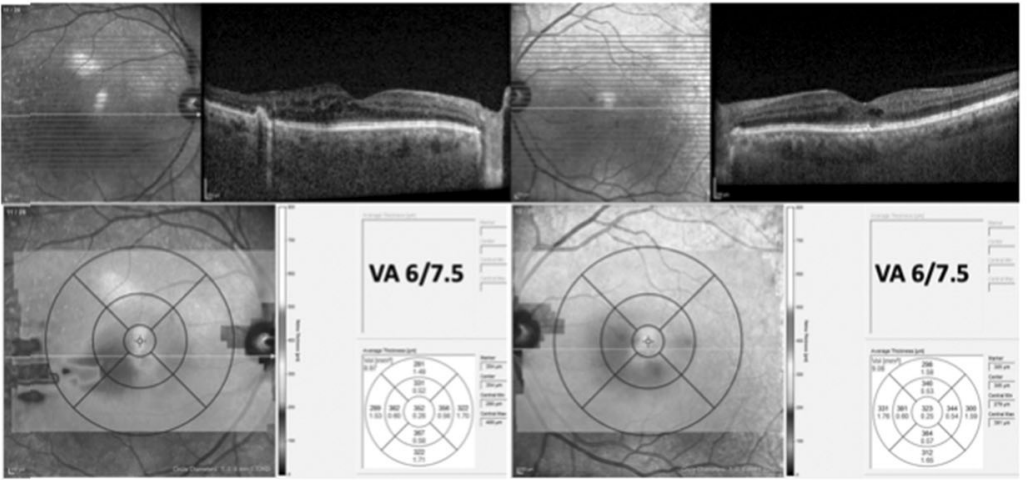

Follow-up Examination - August 2019
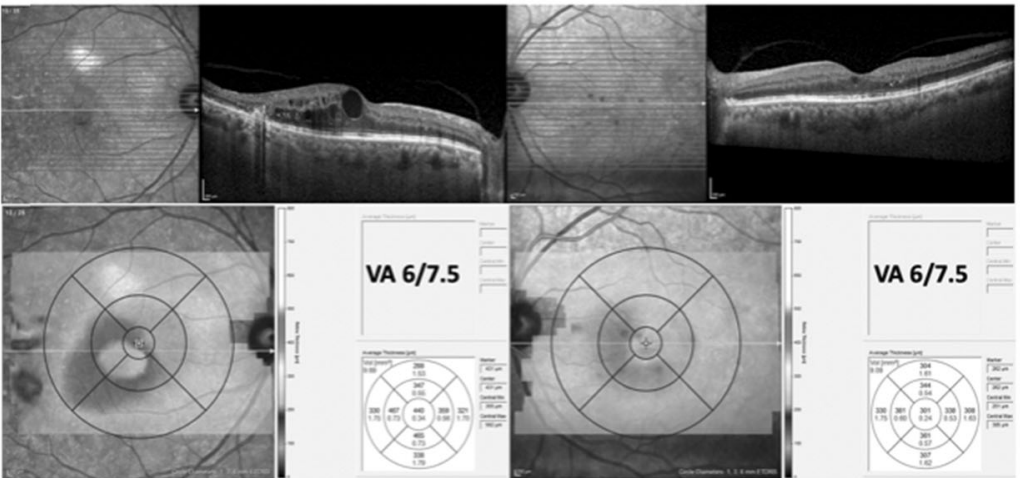

DMO had good vision (visual acuity 20/20) [15]. From a global standpoint, we could estimate that about 11 million adults with DMO and good vision (40\% of 27 million) would need nothing more than observation, according to the DRCR Protocol V. In addition, about two-thirds of these patients may never require anti-VEGF therapy. These 


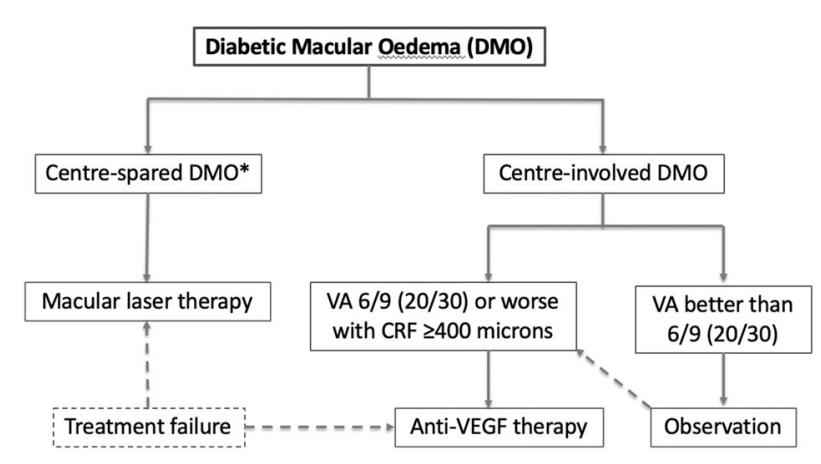

Fig. 2 Management of Diabetic Macular Oedema (DMO)-flowchart showing treatment decision tree for DMO based on involvement of the central macula and visual acuity (VA); VEGF vascular endothelial growth factor; *Non-centred involved DMO is defined as thickened non-central macular subfields on optical coherence tomography map with either (1) at least 2 subfields with thickness above threshold or (2) at least 1 subfield with thickness of at least 15 microns above threshold [16]. CRT central retinal thickness.

results are much needed and welcome relief to the strain and burden on the already overstretched health economies around the world.

In view of these new data, what should clinicians do for their patients with DMO? There are basically three scenarios (Fig. 2). First, for patients with vision loss due to centre-involved DMO, ocular anti-VEGF therapy should continue to be used. Second, for patients with centreinvolved DMO and good vision, they can be observed initially, and anti-VEGF therapy can be offered when visual impairment occurs. For the patients who are keen to reduce their risk of needing anti-VEGF therapy, macular laser photocoagulation can be considered. Third, for patients with clinically significant macular oedema not affecting the central macula, macular laser can be considered to reduce their risk of vision loss. Nonetheless, it should be noted that the progression rate for non-central to centre-involved DMO is generally low (14\%) within 1 year [16], and therefore, it remains debatable whether prompt macular laser is in fact required for all these patients. Ultimately, although data from clinical trials are helpful, clinical management of DMO should be based on discussion with each individual patient, accounting for other factors such as systemic control, peripheral DR severity (e.g., any proliferative disease), visual symptoms and need, as well as fellow eye status.

Despite the clear importance of the data, there remain several unclear aspects regarding the implications of the Protocol V. First, there is currently a lack of good quality epidemiological data on centre-involved DMO, a diagnosis that requires optical coherence tomography imaging of the macula. Most of the previous population-based studies defined DMO based on fundus photographs. In order to better quantify the global impact of the Protocol V data, precise estimates of the prevalence and incidence of centre-involved DMO based on optical coherence tomography are desirable. Second, the amount of centre-involved DMO in the study was relatively low with the mean thickness being 306, 314 and 314 microns in the three arms at baseline. Only $8 \%$ had a macular thickness of 400 microns or more, which is the NICE guidance cut-off for allowing anti-VEGF treatment in England, based on a cost per quality of life year sub group analysis of pivotal studies [17]. Therefore, whether it is safe to watch patients with more severe DMO (i.e., $>400$ microns) but good vision is uncertain. Third, the Protocol V reported a small but significant effect of prompt laser therapy, as compared with initial observation, on reducing the likelihood of needing subsequent anti-VEGF therapy. Cost-effectiveness analysis might be useful to further elucidate the relevance of this finding. Fourth, the Protocol V did not examine the potential influence of the three management strategies on quality of life. Nevertheless, it might be reasonable to assume that the absence of challenge and stress of the frequent visits and injections associated with anti-VEGF therapy would have a positive impact on the patient's quality of life. This is particularly relevant in diabetic patients, who likely have other systemic comorbidities that may already contribute to their personal burden of hospital visits and healthcare cost. Fifth, another unaddressed question is whether leaving DMO beyond 2 years is safe or whether it would affect the response to treatment later. It is known from the VIVID and VISTA studies, for example, that patients in the laser arm, when given aflibercept, did not gain the same vision as those treated at the beginning of the trial [18]. Lastly, current international clinical guidelines suggest that patients with centre-involved DMO should be referred or reviewed in one month [7], although in England screening guidelines are longer at 13 weeks [19]. As shown in the protocol $\mathrm{V}$, two-thirds of these patients with good vision may remain stable under observation, without needing to initiate anti-VEGF therapy within 2 years. Thus, these patients likely do not require such prompt referral or intensive follow-up (e.g., Fig. 1). Additional studies are required to determine how we can reliably identify these "low-risk" patients, and their optimal follow-up interval.

In summary, the DRCR Protocol V study provides clinicians with greater confidence in conservatively managing their patients with centre-involved DMO and good vision, a relatively common clinical scenario. Patients with this condition can now be reassured that the initial observation without any active treatment is a reasonable choice, and their good vision can be maintained as long as they return for regular follow-up visits. Besides DMO, antiVEGF therapy is also used for other ophthalmic indications. The Protocol V data may serve as a sober reminder that it is important for clinicians to be mindful of using this treatment judiciously for the right reason and the right patient. 


\section{Compliance with ethical standards}

Conflict of interest The authors declare that they have no conflict of interest.

Publisher's note Springer Nature remains neutral with regard to jurisdictional claims in published maps and institutional affiliations.

\section{References}

1. Ogurtsova K, da Rocha Fernandes JD, Huang Y, Linnenkamp U, Guariguata L, Cho NH, et al. IDF Diabetes Atlas: global estimates for the prevalence of diabetes for 2015 and 2040. Diabetes Res Clin Pract. 2017;128:40-50.

2. Diabetes facts and stats: 2015. Diabetes UK; 2015. https://www. diabetes.co.uk/diabetes-prevalence.html. Accessed 10 Dec 2019.

3. Cheung N, Mitchell P, Wong TY. Diabetic retinopathy. Lancet. 2010;376:124-36.

4. Tan GS, Cheung N, Simo R, Cheung GC, Wong TY. Diabetic macular oedema. Lancet Diabetes Endocrinol. 2017;5:143-55.

5. Cheung CM, Wong TY. Targeting the effect of VEGF in diabetic macular edema. N. Engl J Med. 2015;373:479.

6. Cheung N, Wong IY, Wong TY. Ocular anti-VEGF therapy for diabetic retinopathy: overview of clinical efficacy and evolving applications. Diabetes Care. 2014;37:900-5.

7. Wong TY, Sun J, Kawasaki R, Ruamviboonsuk P, Gupta N, Lansingh VC, et al. Guidelines on diabetic eye care: the International Council of Ophthalmology recommendations for screening, follow-up, referral, and treatment based on resource settings. Ophthalmology. 2018;125:1608-22.

8. Schmidt-Erfurth U, Garcia-Arumi J, Bandello F, Berg K, Chakravarthy U, Gerendas BS, et al. Guidelines for the management of diabetic macular edema by the European Society of Retina Specialists (EURETINA). Ophthalmologica. 2017;237:185-222.

9. Gale R, Scanlon PH, Evans M, Ghanchi F, Yang Y, Silvestri G, et al. Action on diabetic macular oedema: achieving optimal patient management in treating visual impairment due to diabetic eye disease. Eye. 2017;31 S1:S1-S20.

10. Cheung N, Lam DS, Wong TY. Anti-vascular endothelial growth factor treatment for eye diseases. Br Med J. 2012;344:e2970.

11. Ting DSW, Wong TY. Proliferative diabetic retinopathy: laser or eye injection? Lancet. 2017;389:2165-6.

12. American Society of Retina Specialists. Preferences and trends survey 2014. Chicago, IL: American Society of Retina Specialists; 2014.

13. Egan C, Zhu H, Lee A, Sim D, Mitry D, Bailey C, et al. The United Kingdom Diabetic Retinopathy Electronic Medical Record Users Group, Report 1: baseline characteristics and visual acuity outcomes in eyes treated with intravitreal injections of ranibizumab for diabetic macular oedema. Br J Ophthalmol. 2017;101:75-80.

14. Baker CW, Glassman AR, Beaulieu WT, Antoszyk AN, Browning DJ, Chalam KV, et al. Effect of initial management with aflibercept vs laser photocoagulation vs observation on vision loss among patients with diabetic macular edema involving the center of the macula and good visual acuity: a randomized clinical trial. JAMA. 2019;321:1880-94.

15. Early treatment diabetic retinopathy study design and baseline patient characteristics. ETDRS report number 7. Ophthalmology. 1991;98 Suppl 5:741-56.

16. Friedman SM, Almukhtar TH, Baker CW, Glassman AR, Elman MJ, Bressler NM, et al. Topical nepafenec in eyes with noncentral diabetic macular edema. Retina. 2015;35:944-56.

17. Ranibizumab for treating diabetic macular oedema: technology appraisal guidance [TA274]. 2013. https://www.nice.org.uk/guida nce/ta274.

18. Heier JS, Korobelnik JF, Brown DM, Schmidt-Erfurth U, Do DV, Midena E, et al. Intravitreal aflibercept for diabetic macular edema: 148-week results from the VISTA and VIVID studies. Ophthalmology. 2016;123:2376-85.

19. Preferred practice guidance: diabetic retinopathy screening (DRS) and the ophthalmology clinic set up in England. 2010. https://www.amedeolucente.it/pdf/diabetic-retinopathy-screening. pdf. 